A N N A L E S Annales de Bretagne et des Pays de l'Ouest

Anjou. Maine. Poitou-Charente. Touraine

114-4 | 2007

Varia

\title{
In memoriam Michel DeNIS (1931-2007)
}

\section{Christian Bougeard}

\section{(2) OpenEdition \\ Journals}

\section{Édition électronique}

URL : http://journals.openedition.org/abpo/451

DOI : $10.4000 / a b p o .451$

ISBN : 978-2-7535-1508-6

ISSN : 2108-6443

Éditeur

Presses universitaires de Rennes

\section{Édition imprimée}

Date de publication : 30 décembre 2007

Pagination : 7-9

ISBN : 978-2-7535-0598-8

ISSN : 0399-0826

Référence électronique

Christian Bougeard, «In memoriam Michel DenIS (1931-2007) », Annales de Bretagne et des Pays de l'Ouest [En ligne], 114-4 | 2007, mis en ligne le 30 décembre 2009, consulté le 21 avril 2019. URL:

http://journals.openedition.org/abpo/451; DOI : 10.4000/abpo.451

Ce document a été généré automatiquement le 21 avril 2019

(c) Presses universitaires de Rennes 


\title{
In memoriam Michel DENIS (1931-2007)
}

\author{
Christian Bougeard
}

L'historien Michel Denis vient de disparaître. Né à Rennes en 1931 d'un père ouvrier des chemins de fer et d'une mère agent de service, il a formé des générations d'élèves aux lycées de Laval (1955-1959) puis de Rennes, d'étudiants à la faculté des lettres de Rennes (à partir de 1961), à l'université de Haute-Bretagne (Rennes 2) et à l'Institut d'Études Politiques de Rennes (à partir de 1991). Ses qualités exceptionnelles de pédagogue, sa passion pour l'histoire contemporaine, son intérêt pour l'histoire du temps présent ont orienté de nombreux élèves vers le professorat et la recherche historique. Nombre de travaux : du simple mémoire de maîtrise à la thèse de doctorat d'état ont été menés a bien sous sa direction bienveillante. Michel Denis était aussi un historien dans son temps, un homme et un citoyen engagé dans les combats pour la justice sociale, pour la modernisation de l'université, pour la diffusion du savoir universitaire en direction de tous. Étudiant, élu président de l'AGER (Association générale des étudiants rennais), il avait milité en pleine guerre froide au sein du courant minoritaire et de gauche de l'UNEF contre la majorité corporatiste et de droite, animée en droit par Jean-Marie Le Pen. Jeune professeur agrégé (en 1955), Michel Denis a soutenu les luttes pour la décolonisation, contesté le carcan rigide de l'institution universitaire s'affirmant comme l'un des principaux acteurs de la scène rennaise en mai et juin 1968. Sensible aux mouvements contestataires, il s'est battu pour moderniser l'université en développant la démocratie interne ainsi que de nouvelles formations, notamment lors de sa présidence de l'université Rennes 2 de 1976 à 1980, dans un contexte particulièrement difficile (grèves étudiantes, asphyxie financière, politique de rétorsion de la ministre Alice Saunier-Seïté). C'est d'ailleurs en plein accord avec les instances de l'université que Michel Denis démissionne avec fracas de son poste de président de Rennes 2 le 15 septembre 1980. Fidèle à ses convictions, refusant une réforme qui remet en cause les avancées arrachées en Mai 1968, l'historien s'explique dans un texte adressé au Monde: «Non, je ne restaurerai pas le mandarinat ». 
2 L'œuvre de Michel Denis est importante et multiforme allant du XVIII ${ }^{\mathrm{e}}$ siècle à la Seconde Guerre mondiale. C'est d'abord, en collaboration avec Pierre Goubert, 1789 : les Français ont la parole. Cahiers des États-Généraux, le premier volume de la collection « Archives » en 1964 et un manuel avec Noël Blayau, Le XVIII siècle chez A. Colin en 1970. Le chantier de la Révolution Française est repris à l'occasion du bicentenaire avec Rennes. Berceau de la liberté. Révolution et démocratie : une ville d'avant-garde, éditions Ouest-France, 1989. Mais c'est l'histoire du XIX ${ }^{\mathrm{e}}$ siècle qui est son terrain de prédilection. Michel Denis a publié ses deux premiers articles de chercheur dans les Annales de Bretagne : « La campagne de César contre les Vénètes » en 1954 et « Grandeur et décadence d'une forêt : Paimpont du XVI ${ }^{\mathrm{e}}$ au XIX ${ }^{\mathrm{e}}$ siècle » en 1957, et au moins six articles dans cette revue ainsi que des comptes rendus de lecture.

3 Une partie importante des travaux de Michel Denis a été consacrée à l'étude de la société mayennaise, à ses rapports sociaux, à ses luttes politiques et religieuses, de sa première thèse L'Église et la République en Mayenne de 1896 à 1906, Klincksieck, 1967, à sa thèse d'État, Les Royalistes de la Mayenne et le monde moderne, XIX ${ }^{e}-\mathrm{XX}^{e}$ siècles, Klincksieck, 1977. Connaisseur incomparable du monde des élites de la France de l'Ouest, il s'interroge sur les voies de la modernisation dans les régions qui sont sous l'emprise des hobereaux et des milieux conservateurs, soulignant leurs efforts pour promouvoir un mode de développement, rural et paternaliste, opposé à celui de la bourgeoisie libérale urbaine, pour mieux maintenir leur contrôle social et politique sur la paysannerie. Michel Denis écorne le modèle weberien des cheminements vers la modernité. Cet homme de gauche s'affirme comme un spécialiste de l'histoire de la droite ou plus exactement de L'Histoire des droites en France : il participe d'ailleurs à cet ouvrage collectif dirigé par Jean-François Sirinelli publié chez Gallimard en 1992, en écrivant le premier chapitre du tome 1. Politique, "1815-1848. Que faire de la Révolution française?» En outre, le professeur Michel Denis impulse des travaux sur l'histoire de la Bretagne en dirigeant mémoires et thèses, il siège dans les jurys, donne de nombreuses préfaces ou postfaces, et dirige avec d'autres collègues la publication de colloques ou d'ouvrages dont L'Affaire Dreyfus et l'opinion publique en France et à l'étranger, PUR, 1995, L'Ouest-Éclair. Naissance et essor d'un grand quotidien régional, PUR, 2000 et récemment une Histoire de Rennes, Apogée-PUR, 2006. En 1995, il avait donné une analyse de « l'approche régionale » de la recherche historique dans L'Histoire et le métier d'historien en France, un ouvrage dirigé par François Bédarida. Lors d'une retraite fort active, en conférences et en écrits, Michel Denis a contribué à plusieurs œuvres collectives comme le monumental Dictionnaire du patrimoine breton dirigé par Alain Croix et Jean-Yves Veillard, Apogée, 2000, et il a écrit en collaboration avec Claude Geslin, La Bretagne des Blancs et des Bleus, 1815-1880, une synthèse publiée aux Éditions Ouest-France.

4 Sensibilisé à la question des langues et des cultures régionales, Michel Denis a mené de front le combat pour la reconnaissance du fait régional breton dans des institutions culturelles régionales en construction, comme président du Conseil Culturel de Bretagne (1978-1982), du Conseil national des langues et cultures régionales (1985-1988), ou comme membre du Conseil économique et social, et la réflexion sur les identités régionales à la fin du $\mathrm{Xx}^{\mathrm{e}}$ siècle. Au milieu des années 1970, il avait osé s'attaquer en historien lors d'un colloque à Strasbourg Régions et régionalismes en France, PUF, 1977, à la question controversée de l'attitude du mouvement breton sous l'Occupation : « Mouvement breton et fascisme. Signification de l'échec du second emsav» permettant, sans concessions, d'historiciser un débat occulté ou traité de manière partisane. Et, c'est tout naturellement 
que lors du colloque de Brest sur Bretagne et identités régionales pendant la Seconde Guerre mondiale, CRBC-UBO, 2001, nous lui avions demandé de dresser «un bilan» du « mouvement breton pendant la guerre » à la lumière des travaux les plus récents. Cette communication remarquée confirmait les analyses élaborées un quart de siècle auparavant, et permettait avec d'autres de «refroidir » les polémiques du moment qui faisaient rage par journaux interposés, en remettant à sa place la fraction d'extrême droite et ultra minoritaire du PNB, celle d'un «mouvement breton" dévoyé dans «l'Europe nouvelle» sous la botte nazie et rejeté par les Bretons. Dans un cours public prononcé à l'université Rennes 2 en 2000, Michel Denis avait été amené à s'interroger sur le thème: «L'identité bretonne, identité modèle pour le 21 ${ }^{\mathrm{e}}$ siècle?" (Bretagne 2100. Identité et avenir, coordonné par Alain Croix, PUR, 2001). L'historien, le citoyen, l'acteur du changement de l'université à Rennes et en Bretagne ouvrait des perspectives de réflexion stimulantes.

5 Ceux qui ont été formés par Michel Denis, ceux qui ont travaillé avec lui, ont pu apprécier sa chaleur humaine, sa disponibilité, son charisme. Ils savent bien tout ce qu'ils lui doivent. 